\title{
Lorsque la traduction sert de frontière entre deux cultures : une analyse traductologique de la voix-over dans la version anglaise de Léolo
}

Arianne DesRochers

Université d'Ottawa

La traduction audiovisuelle est un champ émergent au sein de la grande discipline de la traductologie qui attire de plus en plus l'attention des chercheurs, et pour cause : « prominence given to audiovisual productions in today's society makes them an ideal and powerful vehicle for the transmission ... of assumptions, moral values, commonplaces, and stereotypes » (Díaz Cintas 281).La recherche sur la traduction de produits audiovisuels est en effet considérée comme l'une des branches de la discipline les plus en vogue, comme l'explique Maria Tymoczko dans son article « Trajectories of Research in Translation Studies » en 2005. Selon elle, les nouvelles technologies font en sorte que la traduction de produits qui coordonnent du texte et des images (films, publicités, etc.) deviendra l'un des sujets d'études majeurs en traductologie. Tymoczko et le spécialiste de la traduction audiovisuelle Jorge Díaz Cintas s'entendent ainsi pour dire que ce type précis de traduction est de plus en plus paradigmatique et que nous faisons maintenant face à un besoin urgent de recherche en la matière. La traduction de films, par son aspect culturel hautement complexe, peut en dire long sur, par exemple, les habitudes de consommation de cinéma d'une société donnée, ou encore sur les relations entre deux cultures, ce qui en fait un sujet d'étude des plus fascinants.

Dans cet ordre d'idées, un spécimen d'analyse intéressant est le film québécois Léolo, qui constitue l'un des rares exemples où un produit cinématographique est adapté vers l'anglais - donc, d'une communauté linguistique minoritaire, " périphérique ", vers une langue dominante. L'analyse traductologique d'un film requiert bien sûr la participation d'autres disciplines, comme les études cinématographiques, ce qui souligne le caractère interdisciplinaire inhérent à la recherche en traduction audiovisuelle. Le présent article tente, à l'aide du concept de voix-over au cinéma, de poser un regard critique sur le doublage de la narration dans Léolo. Après une brève présentation de ce chef d'œuvre cinématographique, un petit saut en études du cinéma permet de mieux comprendre les rouages de ce qu'on appelle la voix-over. Puis, une mise en lumière du flou 
terminologique entourant cette même notion en traductologie aide à mieux saisir l'objet d'étude, ce qui mènera ensuite à l'analyse de l'accent dans la version anglaise de Léolo et, pour terminer, à une interprétation idéologique de la traduction du film dans son contexte politique. L'analyse de la voix-over de Léolo en traduction anglaise que nous proposons se veut un pas de plus dans l'étude critique, culturelle et idéologique de produits audiovisuels qui voyagent entre une culture dite dominante, l'Amérique du nord anglophone, et une culture périphérique, le Québec francophone.

\section{Léolo}

Sorti en 1992, Léolo est un film québécois réalisé par Jean-Claude Lauzon qui met en vedette Maxime Collin, Ginette Reno, Gilbert Sicotte ainsi que de nombreuses autres grandes pointures du cinéma québécois. Considéré comme l'un des 100 meilleurs films de tous les temps par le Times Magazine en 2005, il gagne également moult prix à sa sortie, dont celui du meilleur scénario original aux Prix Génie, celui du meilleur film canadien au Festival international du film de Toronto et celui du meilleur scénario canadien au Festival international du film de Vancouver, sans oublier qu'il fait partie de la Sélection officielle du Festival de Cannes en 1992. Léolo est aujourd'hui sans contredit considéré comme l'un des chefs d'oeuvre du cinéma québécois.

La pièce maitresse du regretté Jean-Claude Lauzon est un film qui ne se raconte pas. Léolo est une œuvre touchante et troublante d'une grande beauté sur le rêve, l'exutoire, l'enfance, mais également sur la société québécoise et sur la vie de Lauzon - c'est, en effet, un film à teneur autobiographique. Le film suit le parcours de Léo Lauzon, un jeune garçon québécois issu de la classe ouvrière qui grandit dans les années 1950, au moment de la « grande noirceur », et qui rêve de s'échapper de sa triste réalité et de sa famille de malades mentaux. Il invente toutes sortes d'histoires qui lui permettent de rêver, dont celle selon laquelle il serait d'origine italienne. Il imagine, dans la scène la plus célèbre du film, que sa mère a été contaminée en tombant dans un bac de tomates italiennes au marché. Il prétend ainsi être d'origine italienne et souhaite se faire appeler Léolo Lozone. On suit donc, tout au long du film, le parcours imaginaire de ce jeune garçon qui rêve et qui vogue au beau milieu d'une famille malade et d'une réalité incurable, en quête d'amour et d'espoir. 
Léolo est un film riche en références culturelles québécoises, particulièrement en références littéraires, notamment à Réjean Ducharme. Le film est en effet inspiré de L'avalée des avalés, grand roman québécois dont on sent l'influence de l'univers de l'enfance et des jeux de mots dans Léolo. Le film dresse toutefois un portrait sombre et angoissant du Québec : pauvreté, ignorance, maladie mentale et obsession sont les thèmes principaux de l'univers narratif. Jean-Claude Lauzon ne craint pas d'aborder des thèmes durs et délicats, voire tabous : je pense entre autres à la scène où Ti-cul Godin, qui s'apprête à violer un chat à la suite d'une gageure, est présenté comme un jeune adolescent troublé, qui «s'enfile tout ce qui bouge», à " la pissette dévorée par les bactéries » et qui " se prostitue avec son coach de hockey » (Léolo). Le peuple québécois est donc dépeint par Lauzon dans Léolo de façon peu glorieuse : tous les personnages baignent dans l'ignorance et sont condamnés à ne jamais y échapper, y compris Léolo.

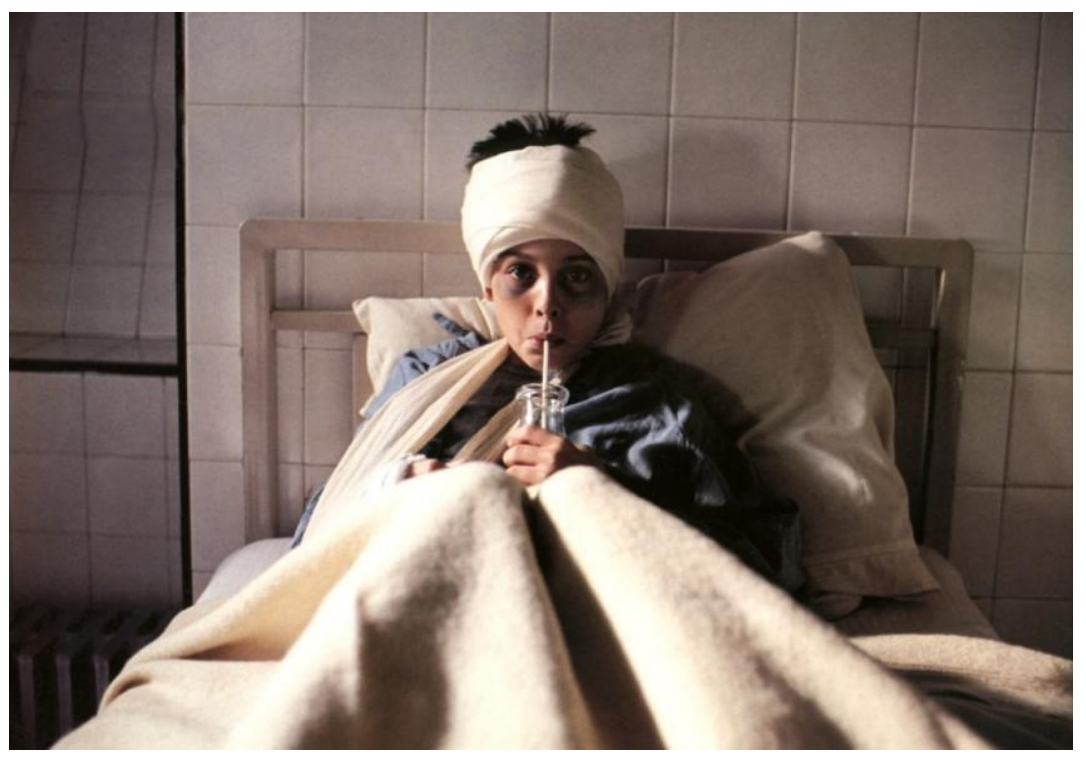

\section{Léo Lauzon}

Mis à part le scénario en tant que tel, c'est-à-dire les dialogues, Léolo est caractérisé par un narrateur omniscient. Une voix narrative, à l'extérieur de la diégèse du film, lit en effet ce qui semble être des extraits du journal intime du jeune Léolo. En version anglaise, les dialogues sont sous-titrés, tandis que la narration (ou voix-over) est doublée en anglais. La voix-over anglaise dans Léolo est un objet d'étude fascinant pour des raisons que nous 
verrons plus loin. Mais avant toute chose, en quoi consiste véritablement cette notion complexe de voix-over, ou voix narrative, au cinéma?

\section{Narration, voix et déliaison au cinéma}

L'emploi de la notion de voix narrative au cinéma, ou voix-over, comme objet d'étude en traductologie exige la participation d'autres disciplines, particulièrement des études cinématographiques. Bien que la parole orale semble avoir été passablement négligée dans les écrits théoriques sur le cinéma (Boillat 27), l'expert en termes de voix au cinéma est sans contredit Michel Chion, qui a beaucoup publié sur la relation audio-visuelle au cinéma dans les années 1980. Ses travaux, toujours fort influents aujourd'hui, ont formé de solides assises théoriques sur lesquelles se basent des études plus récentes, comme celle menée par Alain Boillat dans le cadre de sa thèse de doctorat, publiée sous forme de livre intitulé $D u$ bonimenteur à la voix-over en 2007. Les écrits de ces deux théoriciens composent ainsi un cadre théorique qui permet une analyse éclairée du phénomène de la voix narrative (voix-over) d'un point de vue traductologique. Dans un souci de définition de l'objet d'étude, nous faisons donc appel aux travaux de Chion et de Boillat et aux concepts des études cinématographiques afin de découvrir la nature, les caractéristiques et les rôles de la voix-over au cinéma.

Dans La voix au cinéma, Michel Chion qualifie d'acousmatique un son que l'on entend sans voir son origine (Chion26). C'est précisément le cas de la voix narrative dans Léolo, et de la voix-over de façon plus générale : des paroles sont entendues sans que l'on puisse en visualiser la source à l'écran. La voix narrative n'est perçue qu'oralement, puisqu'elle n'est accompagnée d'aucun support visuel ; on parlera donc d'écoute nonvisualisée. La voix acousmatique est à la fois hors-champ, donc, pour le spectateur, en dehors de l'image, et à la fois dans l'image de derrière laquelle elle provient, comme si elle " rôdait à la surface, à la fois dehors et dedans, en peine d'un lieu où se fixer. Surtout quand on n'a pas encore montré quel corps habite normalement cette voix » (Chion28).C'est exactement le cas du narrateur dans Léolo, qui n'est jamais dévoilé visuellement à l'audience. Chez Chion, l'acousmêtre (l'être acousmatique, que l'on entend sans voir) n'a rien de moins que des pouvoirs magiques : " être partout, tout voir, tout savoir, tout pouvoir. Autrement dit ubiquité, panoptisme, omniscience et toute-puissance » (Chion29). La voix acousmatique entretiend rait également une relation de pouvoir et de 
possession avec l'image. Chion compare ainsi l'acousmêtre à une instance quasi divine, aux limites dont nous ne savons rien.

Quelque vingt-cing ans plus tard, Alain Boillat poursuit la réflexion sur l'acousmêtre dans son livre $D u$ bonimenteur à la voix-over. Il tente une catégorisation des différents types de sons acousmatiques, du point de vue de la non-visualisation de leur source, et conclut qu'il y a trois grandes catégories qui correspondent à la tripartition traditionnelle des anglophones : voix-in, voix-off, et voix-over :

la voix in est perçue comme l'émanation d'un individu visible à l'image en tant que source; la voix off est associée par le spectateur à un locuteur situé dans l'univers diégétique, mais hors champ; la voix-over est quant à elle rapportée à un énonciateur absent, en tant que source verbale, du monde du film. (Boillat 23)

C'est donc sans aucun doute la troisième qui nous intéresse dans le cas de la voix narrative dans Léolo. Pour Boillat, la voix-over plane, comme un esprit, au-dessus du monde diégétique du film. Elle constituerait également le «corps» du film, par la cohérence qu'elle confère à un ensemble fragmentés de plans. Boillat défend que la voixover remplisse une fonction centrale d'instauration de l'univers, puisqu'elle donne accès au monde de la fiction.

On ne se surprend donc pas que Jean-Claude Lauzon ait profité des grands pouvoirs de l'acousmêtre pour mettre sur pied l'univers de Léolo. En un premier temps, l'usage d'un narrateur, qui instaure automatiquement un univers fictif, renvoie directement à la voix narrative en littérature, l'une des sources d'inspiration principales du réalisateur. Puis, la voix ainsi que la parole jouent un rôle crucial dans l'univers de Léolo, qui n'a jamais vu personne dans cette maison lire un livre : «À la maison, je n'avais jamais vu personne lire, ou écrire » (Léolo). Pour le jeune garçon, tout se passe et se transmet plutôt par l'oralité. La narration d'extraits de son journal intime ferait donc office de pont entre son monde et le vrai monde, par le truchement de la parole orale. La voix narrative, ou voix-over, joue donc un rôle de premier plan dans un film comme Léolo. Elle contrôle et possède l'image en même temps qu'elle met en place à elle seule l'univers diégétique du film. La voix-over étant un élément des plus importants du film Léolo, il est intéressant d'examiner sa traduction et son doublage en anglais. 


\section{La notion de voix-over en traductologie}

À la conceptualisation de la voix-over en études cinématographiques s'ajoutent quelques écueils terminologiques propres aux études en traduction. En effet, puisque la traduction audiovisuelle est un champ relativement récent de notre discipline, on observe un certain flou et un manque de consensus autour des définitions de concepts clés, comme celui de voix-over. Selon Luyken, « $[\mathrm{r}]$ evoicing is the superordinate term used to describe the various means of rendering a translated voice track: lip-synch dubbing, voice-over, narration and free commentary » (Luyken et al. dansLittau\&Kuhiwczak124). On constate qu'il y a bel et bien différenciation entre voice-over et narration dans cette citation. Qu'est-ce qui est quoi exactement, du point de vue des études en traduction?

Dans leur ouvrage Voice-Over Translation: An Overview (2010), Eliana Franco, Pilar Orero et Anna Matamala soulèvent le flou terminologique entourant le terme voice-over. Elles donnent d'abord une définition très large du terme : " a narrative technique in which the voice of a faceless narrator is heard over different images and this for different purposes »(Franco, Matamala \& Orero 18), mais s'entendent avec les théoriciens d'études cinématographiques pour mettre l'accent sur la non-visualisation de la source : « all the speech sequences when the narrator cannot be seen » (18). Toutefois, elles constatent que, dans les écrits en traductologie, le terme voice-over est défini et conceptualisé à toutes les sauces. En effet, lorsque Mona Baker parle de « category of revoicing », Fawcett parle de « a type of dubbing », et tandis que pour Gambier on parle plutôt de demi-doublage, pour d'autres on a affaire à " a type of narration », « recorded commentary » ou encore « spoken translation » (voir Franco, Matamala \& Orero 30). Et bien que les auteures de Voice-Over Translation: An Overview ne parviennent pas véritablement à proposer une définition claire, elles n'étudient qu'une seule variante de la voix-over : celle où ce terme désigne le procédé de traduction dans divers programmes télévisés. Leur ouvrage se penche en effet sur la traduction d'une bande sonore originale par une voix-over superposée à la bande son d'origine. C'est le cas, entre autres, des émissions de télé-réalité, ou encore des bulletins de nouvelles, où la voix-over en langue d'arrivée est entendue par-dessus les voix d'origine, que l'on entend toujours mais dont le volume est grandement diminué afin de faciliter la compréhension de la traduction en voix-over. 
Ainsi, puisqu'elles ne s'intéressent qu'à ce type précis de voix-over, les auteures déplorent le flou conceptuel engendré par l'interdisciplinarité qu'entretient la traductologie avec les études cinématographiques : « [o]ne of the negative consequences for Translation Studies of the association between voice-over and narration/commentary established in Film Studies is the fact that we end up with the idea that translated commentaries/narration are instances of voice-over translation » (Franco, Matamala \& Orero 39).Selon elles, la traduction de la voix-over dans Léolo serait donc une translated narration, et non pas une voice-over translation. Or, je ne suis pas tout à fait d'accord avec cette affirmation, tout d'abord parce que je ne crois pas qu'un tel flou terminologique soit une conséquence si négative, mais également parce qu'en langue française, cet écueil notionnel est facilement réglé. Effectivement, alors qu'en anglais on parle de voice-over translation pour une pléthore de procédés et de produits, en français, la distinction se fait par une simple reformulation syntaxique. Pour la voice-over translation telle que définie par Franco, Matamala et Orero et qui renvoie au procédé de traduction (on traduit par, avec une voix-over), on parlerait en français d'une traduction voix-over, ou traduction en voix-over. D'un point de vue cinématographique, toutefois, où la voixover narrative est présente avant même la traduction, on parlera tout simplement de la traduction de la voix-over. Dans un cas, on analyse le procédé traductif, et dans l'autre, la nature du «texte » de départ. Voilà pourquoi je crois qu'il est tout à fait possible et raisonnable de mettre à profit la notion de voix-over, si bien conceptualisée chez Chion et chez Boillat au cinéma, dans une analyse traductologique. Dans le cas de la traduction du film Léolo, j'analyserai donc le doublage de la voix-over narrative (ou narration accousmatique).

\section{La voix-over dans la version anglaise de Léolo}

L'aspect le plus frappant de la traduction anglaise de la voix-over de Léolo est la présence d'un accent québécois, en anglais. En effet, la narration est effectuée tout au long du film par un comédien qui parle anglais avec un accent québécois passablement prononcé que nul auditeur ne saurait manquer. On remarque l'accent québécois francophone soi-disant typique tantôt dans les noms et appellations (Madame, Godin, Fernand, etc.), tantôt dans les the, prononcés de, et ainsi de suite. Un tel choix de traduction est pour le moins surprenant, puisqu'il est rare et obsolète de constater la présence d'un accent en doublage (qui, en règle générale, domestique le produit audiovisuel pour se conformer aux 
habitudes linguistiques de la culture d'arrivée), d'autant plus qu'il est bien connu qu'à Montréal, dans les années 1950 (où prend place le film), l'anglais occupait une place prépondérante. Pourquoi, alors, avoir choisi d'utiliser un accent dans la version anglaise?

La question de l'accent en traduction pose un paradoxe intéressant qui divise manifestement théoriciens et praticiens :

[d] espite Luyken's et al. view on the impossibility of reproducing original accent in the target language, such reproduction has been practiced in the UK. The reproduction of original accents in voice-over versions can be tricky because it may, on the one hand, be positively assessed as even more authentic and, on the other hand, be attacked as a form of caricature or fake. (Franco, Matamala \& Orero 29)

Le choix de reproduire un accent en doublage est donc un couteau à double tranchant qui, nous le verrons, soulève une myriade d'enjeux.

Tout d'abord, conserver un accent québécois en anglais comporte certains dangers. Comme souligné ci-haut, il y a le risque inévitable de tomber dans la caricature, voire l'humiliation, surtout dans le contexte canadien et québécois où les chicanes linguistiques sont coutumes. À mon avis, la reproduction de l'accent original en version anglaise est également absurde, puisque le public étranger ne connait pas nécessairement cet accent et risque de trouver étrange la façon dont s'exprime le narrateur, ce qui pourrait éventuellement avoir un impact direct sur la réception du film à l'étranger. Puis, existe-til véritablement un accent proprement « québécois »? À mon sens, l'accent québécois n'est pas un, mais multiple; il varie de région en région, d'époque en époque et même de locuteur en locuteur. Qui plus est, de nombreux québécois sont anglophones, et bon nombre de francophones sont tout à fait aptes à parler un anglais « sans accent ». Bref, en plus de l'éventuel aspect politiquement incorrect de la conservation de l'accent en traduction, celui-ci est discutable vu son absurdité inhérente.

Néanmoins, il devait manifestement y avoir des raisons de conserver l'accent dans la version anglaise de Léolo. Les responsables de la traduction du film ont peut-être voulu reproduire la façon dont Léolo parlerait en anglais. Cette raison est elle aussi absurde, puisque Léolo ne parle tout simplement pas anglais - et, tel que soutenu ci-dessus, s'il 
parlait anglais, il le parlerait peut-être sans accent ou avec un accent différent. De plus, la présence d'un accent francophone québécois dans la voix-over associe directement la narration à Léolo, alors que dans la version originale, le narrateur n'est jamais dévoilé. Ce faisant, on brise les effets, ou pouvoirs, de l'acousmêtre : il n'est plus inconnu ni mystérieux, il devient un narrateur banal et familier sur lequel on peut mettre un visage. Une telle destruction de l'acousmêtre a inévitablement un impact sur l'univers narratif et diégétique du film dans son ensemble.

Ensuite, la reproduction de l'accent québécois en anglais a pu être faite dans un souci d'authenticité, afin de rendre l'essence purement québécoise du film et des personnages en version anglaise. Encore une fois, cet argument est discutable, puisque le reste du film (scénario et dialogues) est conservé en français et sous-titré en anglais - ce qui, à mon avis, est suffisant pour rendre l'essence québécoise du film. Le souci d'uniformité peut aussi être considéré comme une raison plus ou moins valable : des dialogues en français (sous-titrés en anglais) mélangés avec une narration anglaise parfaite auraient pu donner un résultat étrange (quoique la narration avec accent ait donné un résultat plus qu'étrange).

Une chose est certaine, la version anglaise a voulu mettre l'accent (permettez le jeu de mot) le plus possible sur les caractéristiques et sur les aspects exclusivement québécois de Léolo. Comme le souligne Alain Boillat en faisant référence à Jean Châteauvert, " [1]e grain de la voix, comme le dit Châteauvert, constitue une mine d'informations sur l'individu, le spectateur associant à certaines caractéristiques vocales un physique donné, quand bien même celui-ci n'est jamais montré à l'écran » (Boillat24). Donc, la voix-over narrative, selon Boillat, donne au spectateur des indices quant à l'identité du narrateur et quant à ses principales caractéristiques. Voilà qui pourrait expliquer le choix de l'accent dans la version anglaise, la traduction voulant associer le narrateur et, par extension, le film à une identité québécoise.

\section{Une interprétation idéologique selon le contexte politique}

Dans son article «Clearing the Smoke to See the Screen: Ideological Manipulation in Audiovisual Translation »(2012), Jorge Díaz Cintas appelle à une analyse culturelle et idéologique de la traduction audiovisuelle. Il défend que les productions audiovisuelles, en particulier les programmes fictifs comme Léolo, se prêtent parfaitement à une 
approche idéologique puisqu'elles véhiculent énormément d'information culturelle et puisque la matière linguistique ne représente qu'une partie de leur ensemble sémiotique. En tant qu'espace de pratique discursive, les films et autres produits audiovisuels et leur traduction jouent ainsi un rôle important dans l'articulation de concepts culturels, comme, par exemple, la féminité, la masculinité et l'altérité (Otherness) (Díaz Cintas 281). Díaz Cintas souligne donc le rôle de la traduction dans la construction d'identités subjectives ou collectives (Díaz Cintas 285). Notre hypothèse est que la traduction de Léolo, par la reproduction de l'accent québécois en anglais, travaille à la construction d'identités québécoise et canadienne distinctes. En effet, l'accent québécois ici illustre deux possibilités : soit que ce qui se passe dans le film (un portrait social très sombre et angoissé, comme nous l'avons vu précédemment) appartient à l'Autre et n'a rien à voir avec "nous ", c'est à dire le public anglophone canadien, soit que le Québec a voulu apposer sa propre étiquette sur ce chef d'œuvre du cinéma afin d'en faire un produit exclusivement québécois.

Quelques précisions contextuelles s'imposent. Tout d'abord, bien que Léolo ait été en nomination au Festival de Cannes en 1992, c'est un film qui s'est avant tout illustré sur la scène canadienne (aux Festivals de Vancouver et de Toronto et aux Prix Génie). Il est donc censé d'avancer que la traduction anglaise ait été produite pour le public canadien anglophone principalement (et, par extension, pour les États-Unis). Ainsi, une petite mise en contexte de la situation politique de l'époque s'avère fort intéressante : en 1992, une profonde remise en question identitaire et politique a lieu au Québec et au Canada. Au lendemain du fiasco de l'accord du lac Meech, qui voulait amender la Constitution canadienne afin, entre autres, de reconnaitre le Québec comme « société distincte », une vague de souveraineté sans précédent balaie le Québec avec, notamment, la formation du Bloc québécois en 1991 et le deuxième référendum pour la souveraineté en 1995. Le Québec en est à ce moment à affirmer plus que jamais son identité et à défendre qu'il est une société distincte, ce qui, sans grande surprise, ne plait pas beaucoup au Rest of Canada.

Le vocable clé ici est société distincte : si le Québec en 1992 fait une obsession avec cette appellation, et bien la traduction de Léolo au Canada anglais ne fait qu'abonder dans ce même sens : elle vient même lui donner raison. Il n'est pas clair si l'adaptation du film de Jean-Claude Lauzon a été faite au Canada anglais ou au Québec (l'information n'est malheureusement pas accessible), mais dans les deux cas, une interprétation idéologique 
semblable est possible. Tenons d'abord pour acquis que la traduction a été faite au Canada anglais. En gardant un accent étranger, québécois, dans la version anglaise de Léolo, la traduction crée une distance entre l'identité canadienne et anglophone et l'identité québécoise et francophone. Díaz Cintas croit que " [t]he manipulation in voiceover of foreign accents can be used to define the boundary between the native speaking community and its outside and in so doing risks emphasizing stereotypes that tend towards racialization ». (Díaz Cintas 290) Cette frontière créée par la traduction est on ne peut plus claire dans le cas du doublage de Léolo: par la reproduction de l'accent, la traduction appose l'étiquette " Québécois », " étranger », " autre » au film de Jean-Claude Lauzon, puisque le narrateur omniscient ne parle pas comme les membres de la culture réceptrice. Le contexte sociopolitique de l'époque joue donc un grand rôle dans les choix et stratégies de traduction en place dans Léolo: l'accent crée une véritable frontière entre l'identité canadienne et l'identité québécoise. Du fait que le film de Lauzon dépeigne le Québec comme une société triste et sombre au peuple inculte, obsessif et condamné, la reproduction de l'accent québécois en anglais sert de frontière étanche entre l'« autre » quasi morbide de Lauzon et le « nous » (gens normaux et sains) du reste du Canada.

Si, au contraire, l'adaptation a été faite au Québec, le même argument tient la route, mais en direction opposée. En gardant un accent «typiquement » québécois dans la version anglaise, le Québec se serait assuré d'apposer sa propre étiquette sur ce qui est considéré comme l'un des meilleurs films canadiens jamais produits. Léolo étant un véritable chef d'œuvre couronné de succès, l'accent dans sa version anglaise souligne sa provenance ainsi que son appartenance à la société distincte qu'est le Québec. Ce serait ainsi une stratégie pour s'assurer que le public anglophone soit bien conscient qu'il regarde un produit du Québec, et non pas du Canada.

Nous sommes d'avis que cette explication idéologique de l'accent dans la version anglaise de Léolo est valable, puisque la version espagnole (adaptée en Espagne), ne conserve aucun accent dans le doublage. La narration est effectivement doublée en espagnol d'Espagne, sans aucun accent étranger, soi-disant québécois, pour la simple et bonne raison qu'il n'y a aucun besoin de délimiter la frontière entre l'Espagne et le Québec. L'identité culturelle espagnole est effectivement assez loin de celle du Québec pour ne pas avoir à se définir en comparaison avec elle, contrairement à la culture canadienne anglophone, ou vice-versa. 
Il est important de souligner que toutes ces raisons idéologiques n'ont peut-être pas directement influencé les responsables de la traduction anglaise de Léolo lorsqu'est venu le temps de faire les choix discutés plus haut. Nous n'avançons aucunement que l'accent québécois ait été conservé dans la version anglaise au service des raisons idéologiques énumérées ci-dessus ; autrement dit, ça n'était pas nécessairement intentionnel - et, malheureusement, il n'y aura jamais aucun moyen de le savoir. Toutefois, nous croyons que le contexte sociopolitique et les débats identitaires qui avaient lieu au Canada et au Québec au moment de la sortie de Léolo et de sa traduction ont très certainement influencé (peut-être de façon involontaire, certes) les choix relativement au doublage de sa narration. Finalement, ce qui est certain, c'est que l'adaptation de Léolo et surtout l'accent québécois conservé en anglais, ont eu un effet subséquent de frontière entre la culture canadienne et la culture québécoise. Dans ce cas précis, la traduction aurait donc permis aux sociétés impliquées de non pas s'ouvrir à l'autre, mais bien de se refermer sur elles-mêmes, « hence burning those very bridges that it was supposed to build » (Díaz Cintas 291).

\section{Conclusion}

En somme, nous avons vu que la traduction peut jouer le rôle de frontière entre les cultures dans des moments de tensions identitaires et politiques comme ceux observés au début des années 1990 au Québec et au Canada. Le fait qu'on ait préféré conserver un accent québécois plutôt que de respecter les pouvoirs et les rôles acousmatiques de la voix-over dans la construction de l'univers narratif de Léolo en dit long sur l'impact (direct ou indirect) qu'a un contexte sociopolitique précis sur les choix et stratégies d'adaptation de produits culturels comme les films, puisqu'en effet, ici, le politique a manifestement primé sur l'esthétique. Il serait on ne peut plus intéressant de valider l'hypothèse formulée au long de cet article avec l'étude d'autres grands films québécois de la même période traduits en anglais, comme Octobre (Pierre Falardeau, 1994) ou Jésus de Montréal (Denys Arcand, 1989). Alors qu'il est évident que l'adaptation de Léolo nourrit certains stéréotypes à l'égard du Québec, des études de cas supplémentaires pourraient sans aucun doute donner des indices quant au rôle que joue la traduction de films dans les rapports culturels entre le Québec et le Canada. 


\section{RÉFÉRENCES}

Boillat, Alain. Du bonimenteur à la voix-over. Lausanne : Éditions Antipodes, 2007.

Chion, Michel. L'audio-vision, son et image an cinéma. $2^{\mathrm{e}}$ édition. Paris : Armand Colin, coll. Armand Colin Cinéma, 2005.

-----. La voix au cinéma. Paris : Éditions de l'étoile, Cahiers du cinéma, 1982.

Díaz-Cintas, Jorge. "Clearing the Smoke to See the Screen: Ideological Manipulation in Audiovisual Translation”, Meta 57.2 (2012): 279-93.

Franco, Eliana, Matamala, Anna et PilarOrero. Voice-over Translation, An Overview. Bern: Peter Lang, 2010.

Littau, Karin et Piotr Kuhiwczak. A Companion to Translation Studies. Clevedon: Multilingual Matters, 2007. 